\title{
End-to-End and Redirection Delays in IP Based Mobility
}

\author{
Jon-Olov Vatn \\ Department of Teleinformatics Royal Institute of Technology \\ Electrum 204 S-164 40 Kista, Sweden, Email:vatn@it.kth.se
}

Key words: IP, mobility, end-to-end delay, handover, routing

Abstract: Using the Internet as an infrastructure for mobile, real-time communication is an attractive goal as well as a challenging task. The non-optimal routing inherent in several proposed IP mobility schemes makes it harder to meet the requirement of low end-to-end delay. Mobile users may also perceive decreased performance when a handover between to access points is performed. In this study, common IP based mobility support schemes are evaluated according to their impact on the end-to-end delay and their IP level handover performance. Of the schemes considered, Mobile IPv6 [10] shows the best characteristics. Mobile IPv4 with route optimization [11] is also promising, however, some enhancements are suggested.

\section{INTRODUCTION}

Real-time interactive services like telephony have high requirements on low end-to-end delay $\left(T_{e e}\right)$ and low delay variation. Providing this kind of service over the Internet leads to several challenging problems, due to the delay variations inherent in datagram networks. The delay variations can be addressed by buffering at the receiver, but the requirement on low maximum end-to-end delay $\left(T_{e e, \max }\right)$ is still an issue ( $T_{e e, \max }$ is often considered to be around $150 \mathrm{~ms}$, but may be stretched up to $400 \mathrm{~ms}$ for trained users[5]).

When extending these real-time services to include mobile users, it will be even harder to meet the requirement of low $T_{e e}$, since packets may not take the shortest path between the communicating entities due to non-optimal routing, and it is difficult to guarantee an acceptable quality of service in all the locations that the user may move to. Also, 
mobile users may perceive reduced performance when moving between different access points (APs), i.e., performing a handover. Packets inflight to the mobile user's previous point of attachment, may be lost or unacceptably delayed. The time interval when users may perceive reduced performance due to a handover will depend on factors such as movement detection delay, time to acquire a new care-of IP address $[13,14]$, and the time it takes to redirect $\left(T_{r d}\right)$ data to this new location.

The objective of this paper is to compare a set of proposed mobility support schemes and evaluate their performance when the mobile user is visiting a foreign IP subnet (by looking at $T_{e e}$ ), and when it performs a handover between subnets (by looking at $T_{r d}$ ). In section 2 we cover the basic concepts of IP mobility. In section 3 , the different mobility support schemes are presented and analyzed using symbolic expressions for $T_{e e}$ and $T_{r d}$. These results are summarized and discussed in section 4 . Finally, section 5 concerns future work.

\section{IP BASED MOBILITY}

Each node node in the Internet is identified by one (or more) IP address(es), and the IP addresses of nodes on the same IP subnet have a common prefix. This makes routing scalable, since routers only have to keep track of networks, not individual hosts. However, this implies that a node that leaves one IP subnet to attach to another will have to acquire a new address corresponding to the new subnet. Furthermore, the node needs to be identified by same address as before to be able to keep its ongoing higher level connections.

Therefore, Mobile IPv4 (MIPv4)[8] lets the mobile nodes (MNs) be associated with two IP addresses: one home address belonging to a home network, and a care-of address (COA) associated with the visited subnet. When the MN is away from home, a host on the home network, a home agent (HA), intercepts packets destined for the MN and tunnels[9] them to the MN's current COA. This tunnel can end at the MN itself or at a dedicated host, a foreign agent (FA), on the visited subnet. In the former case we call the COA a co-located COA and in the latter case we have a foreign agent COA. When a $\mathrm{MN}$ moves to a new subnet, it needs to inform its HA about its new COA, so that the HA can redirect packets accordingly.

For traffic going in the other direction (upstream), MIPv4 offers two possibilities: the MN can either send data directly to the correspondent node $(\mathrm{CN})$, or tunnel the packets back to its HA, which in turns routes them towards the CN. These two routing schemes are called triangular routing and reverse tunneling, see Figure 1. 


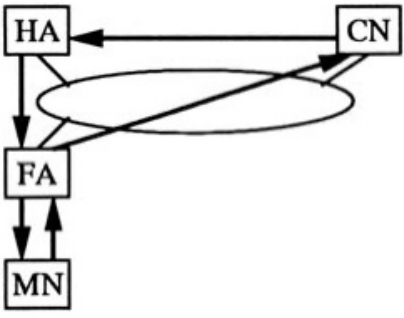

a)

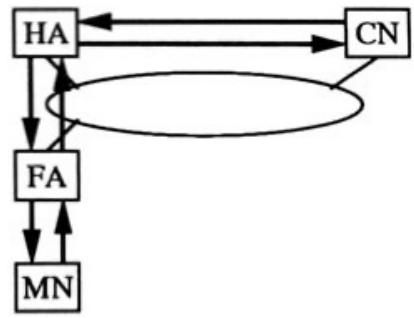

b)

Figure 1 Mobile IPv4 with (a) triangular routing and (b) reverse tunneling.

In the next section, we will analyze MIPv4 and a set of other proposed IP mobility support schemes and evaluate them with respect to their effect on the end-to-end delay $\left(T_{e e}\right)$ and the redirection delay $\left(T_{r d}\right)$. Since these metrics may differ for the downstream (towards the MN) and the upstream (towards the $\mathrm{CN}$ ) flow, we define these delays separately.

Definition 1 Downstream end-to-end delay $\left(T_{e e, d o}\right)$ : The time elapsed from when a CN sends a packet until it arrives at the MN.

Definition 2 Upstream end-to-end delay $\left(T_{e e, u p}\right)$ : The time elapsed from when a MN sends a packet until it arrives at the CN.

Definition 3 Downstream redirection delay $\left(T_{r d, d o}\right)$ : The amount of time when packets are lost (or delayed so that $T_{e e, d o}>T_{e e, \max }$ ) after a MN has acquired a new COA, because the packets were still directed to the old COA.

Definition 4 Upstream redirection delay $\left(T_{r d, u p}\right)$ : The amount of time a $\mathrm{MN}$ is hindered from sending data to its $\mathrm{CN}(\mathrm{s})$ via the new subnet after the MN has acquired a new COA.

The definitions of $T_{e e, d o}$ and $T_{e e, u p}$ are quite straightforward, however, one should note that we do not consider the delay associated with packetization at the sender. If we compare these two metrics for the setup in Figure 1, one can expect that $T_{e e, d o}$ will be equal for triangular routing and reverse tunneling, while $T_{e e, u p}$ will be lower for triangular routing.

Regarding $T_{r d}$, the definitions need some further explanation. We assume that the MN is roaming in area with overlapping cells, but that the MN can only attach to one AP at a time. Then $T_{r d, d o}$ represents the period of time when packets are lost, if the time to shift between the APs and the time to acquire a new COA were both zero. For the upstream traffic the situation is different, since the $\mathrm{MN}$ itself could be 
seen as the point of redirection. Nevertheless, for some of the mobility support schemes the MN may be hindered from sending data to the CN for a certain amount of time $T_{r d, u p}$.

\section{ANALYSIS OF IP MOBILITY SCHEMES}

There exist several proposals for alternatives or extensions to the basic MIPv4 scheme introduced in section 2. The additional schemes we have considered are referred to as Mobile IPv4 with route optimization [11], Mobile IPv4 with Hierarchical Foreign Agents[7] and Mobile IPv6 (MIPv6)[10]. The first two contain extensions to MIPv4 that reduce $T_{e e}$ and $T_{r d}$ respectively. MIPv6 is covered since it will become important when IPv6 deployment takes off.

We will present symbolic expressions for the performance metrics defined in section 2, for each of these schemes. We restrict our analysis to the case were both the $M N$ and the $C N$ are mobile and we let them use the same mobility support scheme. The only difference between the MN and the $\mathrm{CN}$ is that the $\mathrm{MN}$ is performing a handover, while the $\mathrm{CN}$ is staying at a certain location during this period of time.

Sections 3.1-3.4 concern different IPv4 mobility support schemes based on the use of foreign agents, while section 3.5 treats aspects of using colocated COAs in MIPv4. Section 3.6 concerns mobility support in IPv6.

\subsection{MIPV4 WITH TRIANGULAR ROUTING}

As described in section 2, MIPv4 offers two possible routing schemes, triangular routing and reverse tunneling. Here we will present the delays in triangular routing, while reverse tunneling is covered in the next section.

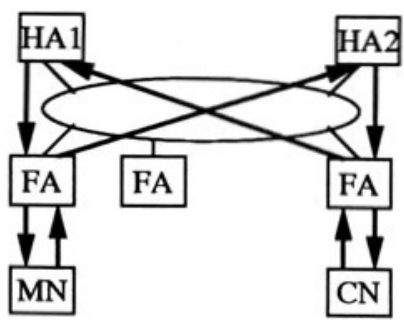

a)

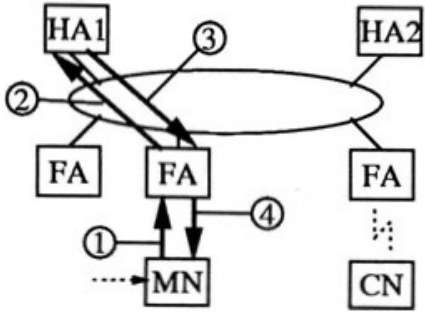

b)

Figure 2 MIPv4 with triangular routing: (a) data paths, and (b) signaling paths.

The data and signaling paths for MIPv4 with triangular routing are shown in Figure 2. Since the CN is also mobile (with home agent HA2), the data path takes the form of a bow-tie $(\bowtie)$ and not a triangle as 
in Figure 1. Symbolic expressions for $T_{e e, d o}$ and $T_{e e, u p}$ are shown in equation 1 and 2.

$$
\begin{aligned}
& T_{e e, d o}=T_{c n->h a 1}+T_{h a 1->m n} \\
& T_{e e, u p}=T_{m n->h a 2}+T_{h a 2->c n}
\end{aligned}
$$

$T_{c n->h a 1}$ is the delay for packets to travel from the CN to the HA of MN, $T_{h a 1->m n}$ is the delay from the HA to MN and so on. Processing delays at intermediate nodes, e.g., HAs and FAs, are not stated explicitly. Instead we emphasize the delay related to the path the packets will traverse. We do not claim that processing delay is irrelevant, however, its impact decreases with improved hardware performance.

To redirect the downstream traffic, the $\mathrm{MN}$ sends a Binding Update $(B i n U p)$ to its HA via the new FA. The HA responds with a Binding Acknowledgment (BinAck) to acknowledge a successful (or unsuccessful) binding update and redirects the packets to the new COA. Packets lost after the MN has acquired the new COA are the ones in-flight between the HA and the previous COA plus the ones arriving at HA while the $B i n U p$ travels from the MN to the HA. Thus, $T_{r d, d o}$ will be the sum of the network delay between the HA and the MN's old and new point of attachment (denoted $T_{h a 1->m n, o l d}$ and $T_{m n->h a 1}$ respectively).

The time to redirect the upstream data flow depends on the roundtrip time from the MN to its HA, i.e, the MN should wait for a (positive) $B i n A c k$ in response to the $B i n U p[8]$ before it updates its routing table in accordance with the new subnet. Symbolic expressions for $T_{r d, d o}$ and $T_{r d, u p}$ are given in equation 3 and 4.

$$
\begin{aligned}
& T_{r d, d o}=T_{h a 1->m n, o l d}+T_{m n->h a 1} \\
& T_{r d, u p}=T_{m n->h a 1}+T_{h a 1->m n}
\end{aligned}
$$

\subsection{MIPV4 WITH REVERSE TUNNELING}

In Mobile IPv4 with triangular routing, the MN uses its home IP address as source address, even if it is currently attached to another network. This may lead to problems, since security conscious routers may drop packets, which do not have a topologically correct source address [3]. One way to address this problem is to let the FA tunnel the packets from the MN to the CN via the HA, as shown in Figure 3. These packets will be able to pass ingress filtering routers, since the source address of the "outer" IP header will be the IP address of the FA. This method is called reverse tunneling[6], since both downstream and upstream traffic are tunneled between the HA and the FA. (Reverse tunneling can also be useful if the MN sends multicast packets, since multicast routing 


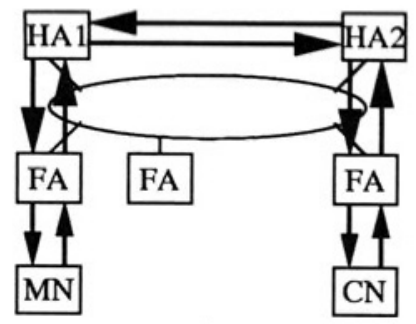

a)

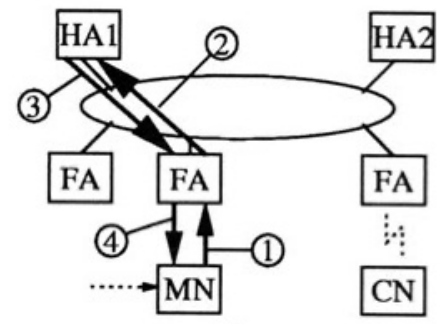

b)

Figure 3 MIPv4 with reverse tunnels: (a) data paths, and (b) signaling paths.

protocols based on reverse path forwarding also depend on topologically correct source addresses.) Symbolic expressions for $T_{e e, d o}$ and $T_{e e, u p}$ are given in equations 5 and 6.

$$
\begin{aligned}
& T_{e e, d o}=T_{c n->h a 2}+T_{h a 2->h a 1}+T_{h a 1->m n} \\
& T_{e e, u p}=T_{m n->h a 1}+T_{h a 1->h a 2}+T_{h a 2->c n}
\end{aligned}
$$

The tumnel from the FA to the HA will not be available before the FA receives a positive $B$ in $A c k$ from the $H A$, thus sending data from the MN before receiving the $B i n A c k$ makes little sense, since the FA is likely to drop them. Hence, if reverse tunneling is used, the $M N$ will not be able to send data packets via the new FA before the BinAck has been received. $T_{r d, d o}$ and $T_{r d, u p}$ will be the same as for MIPv4 with triangular routing, see equation 3 and 4 .

\subsection{MIPV4 WITH ROUTE OPTIMIZATION}

To reduce the end-to-end delays present in MIPv4, there is an IETF draft[11] proposing a scheme for route optimization. This could be seen as an extension to the MIPv4 protocol, where CNs can tunnel packets directly to the MN's current COA, instead of routing them via the HA. When a HA, that supports route optimization, intercepts data destined for one of its MNs, it will tumnel the packets from the CN to the MN (as before), but also send a BinUp to the CN to inform it about the MN's current COA. Thus, a CN will send the first (few) packet(s) via the HA, but is then able tunnel the packets directly to the MN's current location, see Figure 4a). The MN will also tunnel packets (upstream) to the CN, using its home address source address in the "outer" and the "inner" header. $T_{e e, d o}$ and $T_{e e, u p}$ will be low, see equations 7 and 8 .

$$
\begin{aligned}
& T_{e e, d o}=T_{c n->m n} \\
& T_{e e, u p}=T_{m n->c n}
\end{aligned}
$$




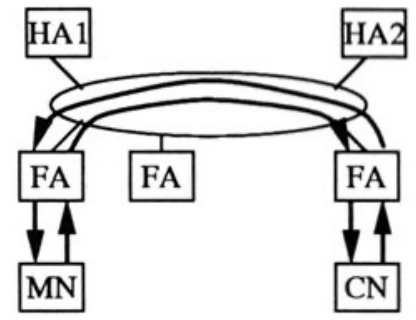

a)

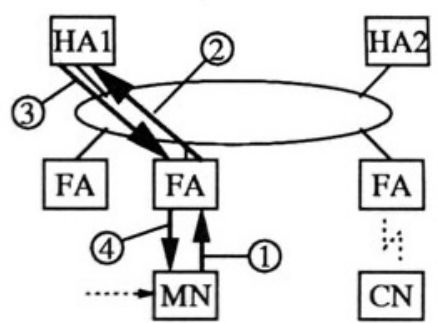

b)

Figure 4 MIPv4 with route optimization: (a) data paths, and (b) signaling paths.

To avoid losing all packets in-flight between the HA and the old FA, the route optimization draft also includes support for smooth handover. The MN can inform its old FA where it should forward packets it receives after the MN has moved. $T_{e e, d o}$ for these packets will be

$$
T_{e e, d o}=T_{c n->f a, o l d}+T_{f a, o l d->m n} \quad(\text { smooth })
$$

The handover procedure differs somewhat from the schemes presented earlier. The MN will inform its HA (and possibly its old FA) about its new COA. The HA will then send a BinUp to inform the CNs, so that they can redirect the downstream packets, see Figure $4 \mathrm{~b}$ ). (BinUps can also be triggered if the HA is notified about CNs with stale mobility bindings by the old FA.) Due to the smooth handover feature, data inflight can be redirected by the old FA, thus decreasing $T_{r d, d o}$ if the new and old FA are relatively close and if $T_{e e, d o}$ in equation 9 is less than $T_{e e, m a x}$. Smooth handover is likely to have most impact when the handover is performed between IP subnets within the same administrative domain (AD), i.e., an intra- $A D$ handover. $T_{e e, u p}$ ought to be the same as for the base MIPv4 scheme, see section 3.1. Hence, the redirection delays will be as follows:

$$
\begin{aligned}
T_{r d, d o} & = \begin{cases}T_{m n->f a, o l d} & \text { intra-AD } \\
T_{c n->m n, o l d} & \\
T_{h a 1->c n} & \text { cross-AD }\end{cases} \\
T_{r d, u p} & =T_{m n->h a 1}+T_{h a 1->m n}
\end{aligned}
$$

Equation 11 assumes that HA1 has cached a binding to the CN's current location. This assumption is reasonable since the CN will regularly contact HA1 to check the validity of the binding it has cached for MN. 


\subsection{MIPV4 WITH HIERARCHICAL FOREIGN AGENTS}

Perkins introduces a mobility support architecture with multiple levels of redirection[7]. The idea is that handovers performed within an AD can be handled locally. This will both reduce the amount of signaling sent over the backbone and lead to faster handovers. FAs are arranged in a hierarchy, where packets destined for the MN arrive at some top level FA. Packets will then be redirected through multiple tunnels until they reach the FA closest to the $\mathrm{MN}$, see Figure $5 \mathrm{a}$ ). When a $\mathrm{MN}$ performs a handover, the $B i n U p$ only needs to travel to the FA that constitutes the lowest common node (the branching point) in the FA hierarchy. If there is no common FA, the BinUp will have to go all the way to the HA. As a hierarchy of FAs are not likely to span multiple ADs, only intra- $A D$ handovers are likely to benefit from this architecture.

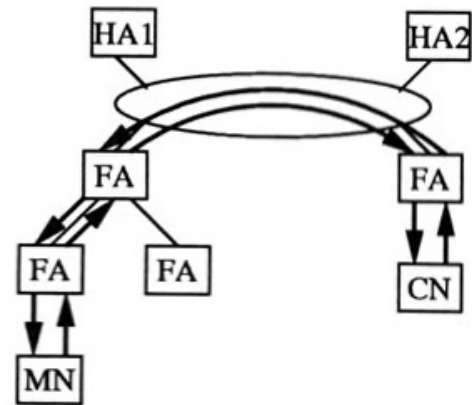

a)

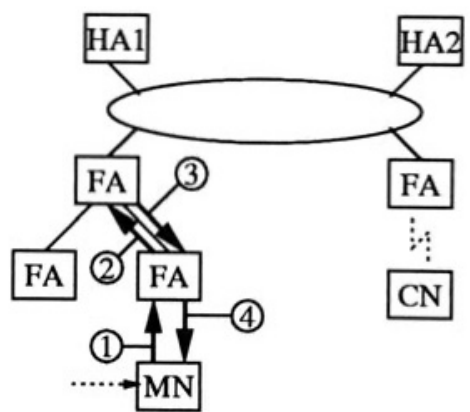

b)

Figure 5 MIPv4 with hierarchical foreign agents: (a) data paths, and (b) signaling paths.

This scheme was designed as an extension of MIPv4; however, schemes based on hierarchical FAs could be combined with several other schemes for cross- $A D$ handover. If we consider the case where hierarchical FAs are combined with the route optimization scheme of section 3.3 (skipping the smooth handover feature for simplicity) $T_{e e}$ and $T_{r d}$ will be as follows:

$$
\begin{aligned}
T_{e e, d o} & =T_{c n->m n} \\
T_{e e, u p} & =T_{m n->c n} \\
T_{r d, d o} & = \begin{cases}T_{f a, b r a->m n, o l d}+T_{m n->f a, b r a} & \text { intra-AD } \\
T_{c n->m n, o l d}+T_{m n->h a 1}+T_{h a 1->c n} & \text { cross-AD }\end{cases} \\
T_{r d, u p} & = \begin{cases}T_{m n->f a, b r a}+T_{f a, b r a->m n} & \text { intra-AD } \\
T_{m n->h a 1}+T_{h a 1->m n} & \text { cross-AD }\end{cases}
\end{aligned}
$$




\subsection{MIPV4 WITH CO-LOCATED CARE-OF ADDRESSES}

Instead of relying on FAs, the MN could acquire a COA of its own, e.g., by use of DHCP[2]. This kind of COA, called a co-located COA, could be used instead of foreign agent COAs in the schemes already presented $^{1}$.

The only significant difference in the metrics we are studying concerns $T_{r d, u p}$. Once the MN has acquired a COA on a new subnet, it should be able to send data to its CNs immediately, since there is no need to wait for a positive BinAck. Thus, $T_{r d, u p}$ is equal to zero for the triangular routing, reverse tunneling and route optimization schemes if co-located COAs are used.

$$
T_{r d, u p}=0 \quad \text { (all MIPv4 schemes) }
$$

The route optimization draft[11] does not consider co-located COAs, but it should work well except for two features that rely on the existence of FAs; smooth handover and stale mobility binding detection, see section 3.3. Since there is no smooth handover support, $T_{r d, d o}$ will be as long for intra-AD as for cross-AD handovers when using MIPv4 with route optimization, see equation 17 (compare with equation 10).

$$
T_{r d, d o}=T_{m n->h a 1}+T_{h a 1->c n}+T_{c n->m n, o l d}
$$

\subsection{MOBILITY SUPPORT IN IPV6}

Work is in progress to design mobility support in IPv6 (MIPv6)[10]. The major difference with respect to MIPv4 is that there are no FAs in MIPv6, i.e., only co-located COAs are used. To get a COA on a new IPv6 subnet should be relatively easy, as the MN has the possibility to use stateless[12] and stateful[1] IPv6 address autoconfiguration.

In MIPv6 a CN is able to send data directly to the MN so $T_{e e, d o}$ and $T_{e e, u p}$ will be the same as for route optimization in MIPv4, see equations 7 and 8.

When the MN has moved to a new IPv6 subnet and acquired a COA on this subnet, it will send a BinUp message ${ }^{2}$ to its HA to register this COA. The MN can also send a BinUp to its $\mathrm{CN}(\mathrm{s})$, and also to a router ${ }^{3}$

\footnotetext{
${ }^{1}$ To use co-located COAs in a hierarchical FA setup would be possible if the MN's are informed about the IP address of the FA above it in the hierarchy. This could be accomplished, e.g., by adding a hierarchical FA option in DHCP.

${ }^{2}$ It does not have to be a separate IPv6 packet, as a binding update destination option can be included in any existing packet being sent to the same destination.

${ }^{3}$ This kind of router needs special support for smooth handover.
} 


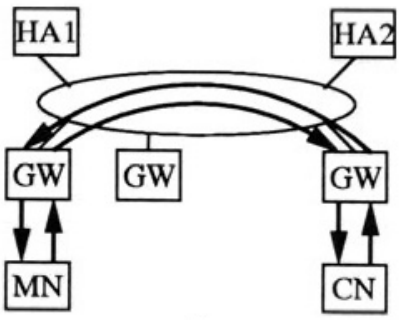

a)

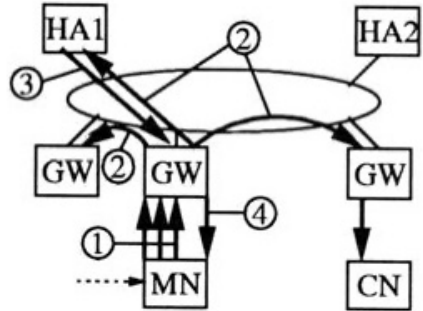

b)

Figure 6 MIPv6 with route optimization: (a) data paths, and (b) signaling paths.

on its previous subnet (for smooth handover). Since the MN can send a Bin $U p$ directly to its $\mathrm{CN}, T_{r d, d o}$ will be shorter, as compared to route optimization in MIPv4 (see Equation 10). $T_{r d, u p}$ will be approximately zero, since the MN can redirect its outgoing flow immediately.

$$
\begin{aligned}
& T_{r d, d o}= \begin{cases}T_{m n->g w, o l d} & \text { intra-AD } \\
T_{m n->c n}+T_{c n->m n, o l d} & \text { cross-AD }\end{cases} \\
& T_{r d, u p}=0
\end{aligned}
$$

Reverse tunneling is also possible in MIPv6. The end-to-end and redirection delays will then be the same as when using co-located COAs with reverse tunneling in MIPv4, see sections 3.2 and 3.5.

\section{RESULTS AND CONCLUSION}

To achieve low end-to-end and redirection delays, we are concerned about the number of times that a data or a signaling packet may have to traverse the backbone network. By use of the symbolic expressions presented in section 3 , we can easily compare the different mobility support schemes. Except for the expressions concerning intra-AD handovers, each component in the expressions constitutes a potential backbone traversal.

- End-to-end delay: It is important to have $T_{e e, d o}$ and $T_{e e, u p}$ less than $T_{e e, m a x}$. As we have assumed that $\mathrm{MN}$ and $\mathrm{CN}$ use the same scheme, $T_{e e, d o}$ and $T_{e e, u p}$ will give the same number of traversals. From the equations in section 3 we can see that:

- MIPv6 and MIPv4 with route optimization gives only one backbone traversal. $(7,8)$

- Triangular routing can give two backbone traversals. (1,2)

- Reverse tunneling can give three backbone traversals. $(5,6)$ 
If the network delays between the involved entities are large, the schemes using route optimization are the only ones that can achieve acceptable performance. Triangular routing and bidirectional tunneling may give long $T_{e e}$ even in the case the MN and the CN are attached to the same subnet.

- Downstream redirection delay: The downstream redirection delay can differ if the handover is intra-AD or cross-AD:

- For intra-AD handovers schemes based on hierarchical FAs or smooth handover are superior, since $T_{r d, d o}$ does not include any backbone traversals in this case. $(14,18)$

- For cross-AD handovers no scheme provides good support. $\mathrm{MIPv} 4$ with triangular routing, MIPv4 with reverse tunneling and MIPv6 (with route optimization or reverse tunneling) all risk losing packets corresponding to the delay of two backbone traversals. $(3,18)$

For MIPv4 with route optimization the situation is even worse, since packets corresponding to three backbone traversals may be lost. (10)

To improve $T_{r d, d o}$ for MIPv4 with route optimization, the obvious approach would be to let the MN send a BinUp directly to the CNs, however, this is currently not suggested in [11].

It should be noted that using hierarchical FAs is of no use for handovers across ADs, unless the hierarchy spans over several domains. Smooth handover mechanism are not likely to help either, since packets will have to traverse the backbone multiple times and possibly arrive to late at the MN.

- Upstream redirection delay: For the upstream redirection delay, we get different results if co-located or foreign agent COAs are used:

- Schemes using co-located COAs have a $T_{r d, u p}$ of zero. $(16,19)$

- $T_{r d, u p}$ for intra-AD handovers with hierarchical FAs will not involve any backbone traversals.(15)

- Otherwise, schemes based on foreign agent COAs, the MN waits for a positive BinAck, leading to a $T_{r d, u p}$ of two backbone traversals. $(4,11)$

It is worth noting that the $\mathrm{MN}$ receives information about an appropriate gateway on the new subnet in the Agent Advertisements sent out by the FA. Hence, it ought to be possible for the MN to 
send data (possibly simulcasting the packets to the old FA as well) to the CN via that gateway at the same time as it is able to send the BinUp, giving a $T_{r d, u p}$ of approximately zero.

The overall conclusion is that MIPv6 is the scheme most well suited for services like IP based mobile telephony, since it gives the "lowest possible" end-to-end delay and relatively low redirection delay. Of the IPv4 schemes, MIPv4 with route optimization is the most promising one, due to its low end-to-end delay. To enhance it even further, the MN should be able to send BinUps to the CNs directly. Furthermore, to be able to cope with ingress filtering routers, support for reverse tunneling towards the CNs may be needed.

\section{FUTURE WORK}

In this study we have assumed that necessary keys for authentication etc has already been exchanged. However, to enable wide spread deployment of IP based mobility, a global key management infrastructure will be needed. This is particularly important for schemes where the MN sends BinUps directly to its CN(s). Related to this is the need for authentication, authorization and accounting (AAA) services[4]. To evaluate how this affects $T_{e e}$ and $T_{r d}$ is an interesting and important extension to this work.

The study could also be extended to give numerical results by measuring network delays for different distances and topologies and use these values in the equations in section 3. Furthermore, one should make measurements on (high performance) implementations of the mobility support schemes to study whether the associated processing delays are negligible or not.

Development of efficient link layer support will affect the results of this study. If the MN is able to connect to several APs simultaneously, the impact of $T_{r d}$ will be reduced. $T_{r d}$ will still be of importance, since it affects the need for cell overlap areas and fast movement detection mechanisms.

\section{References}

[1] J. Bound and C. Perkins. Dynamic Host Configuration Protocol for IPv6 (DHCPv6) <draft-ietf-dhc-dhcpv6-14.txt>, February 1999. Internet draft. Work in progress.

[2] R. Droms. RFC 2131: Dynamic host configuration protocol, March 1997. 
[3] P. Ferguson and D. Senie. RFC 2267: Network ingress filtering: Defeating denial of service attacks which employ IP source address spoofing, January 1998.

[4] S. Glass, T. Hiller, S. Jacobs, and C. Perkins. Mobile IP Authentication, Authorization, and Accounting Requirements < draft-ietfmobileip-aaa-reqs-03.txt>, March 2000. Internet draft. Work in progress.

[5] International Telecommunication Union Telecommunication Standardization Sector (ITU-T). Recommendation G.114, Transmission Systems and Media, General Characteristics of International Telephone Connections and International Telephone Circuits, One-Way Transmission Time, February 1996.

[6] G. Montenegro. RFC 2344: Reverse tunneling for mobile IP, May 1998.

[7] C. Perkins. Mobile-IP Local Registration with Hierarchical Foreign Agents <draft-perkins-mobileip-hierfa-00.txt>, February 1996. Internet draft. Work in progress.

[8] C. Perkins. RFC 2002: IP mobility support, October 1996.

[9] C. Perkins. RFC 2003: IP encapsulation within IP, October 1996.

[10] C. Perkins and D. Johnson. Mobility Support in IPv6 <draftietf-mobileip-ipv6-09.txt>, October 1999. Internet draft. Work in progress.

[11] C. Perkins and D. Johnson. Route optimization in mobile ip < draftietf-mobileip-optim-08.txt>, February 1999. Internet draft. Work in progress.

[12] S. Thomson and T. Narten. RFC 2462: IPv6 stateless address autoconfiguration, December 1998.

[13] J-O Vatn. Long Random Wait Times for Getting a Care-of Address are a Danger to Mobile Multimedia. In 1999 IEEE International Workshop on Mobile Multimedia Communications (Mo$\left.M u C^{\prime} 99\right)$, November 1999. San Diego, CA, USA.

[14] J-O Vatn and G.Q. Maguire Jr. The effect of using co-located careof addresses on macro handover latency. In 14th Nordic Teletraffic Seminar, August 1998. Lyngby, Denmark. 